\title{
On Wireless Network Coverage in Bounded Areas
}

\author{
Zuoming $\mathrm{Yu}^{\dagger *}$, Jin Teng ${ }^{\ddagger *}$, Xinfeng $\mathrm{Li}^{\ddagger}$ and Dong Xuan ${ }^{\ddagger}$ \\ ${ }^{\dagger}$ Dept. of Basic Courses, Jiangsu University of Science and Technology, China. yuzuoming1981@gmail.com \\ ${ }_{\ddagger}^{\ddagger}$ Dept. of Computer Science and Engineering, The Ohio State University, USA. \{tengj, lixinf, xuan\}@cse.ohio-state.edu
}

\begin{abstract}
In this paper, we study the problem of wireless coverage in bounded areas. Coverage is one of the fundamental requirements of wireless networks. There has been considerable research on optimal coverage of infinitely large areas. However, in the real world, the deployment areas of wireless networks are always geographically bounded. It is a much more challenging and significant problem to find optimal deployment patterns to cover bounded areas. In this paper, we approach this problem starting from the development of tight lower bounds on the number of nodes needed to cover a bounded area. Then we design several deployment patterns for different kinds of convex and concave shapes such as rectangles and $L$-shapes. These patterns require only few more nodes than the theoretical lower bound, and can achieve efficient coverage. We have also carefully addressed and evaluated practical conditions such as coverage modeling and connectivity regarding our deployment patterns.
\end{abstract}

\section{INTRODUCTION}

\section{A. Motivation}

How to optimally cover a plane with certain shapes is a classical problem dating back to the Aristotle times. The interest in this problem is revived with the rise of research in wireless networks. In wireless networks, coverage is a critical issue. For example, in cellular networks or wireless local area networks (WLANs), we need to deploy basestations or access points (APs) such that every client in a designated region can be served. In wireless sensor networks (WSNs), sensors are expected to cover the entire monitoring region.

Kershner proved in 1939 that the honeycomb structure, also known as the triangular lattice, is the optimal pattern to cover unbounded areas [12]. Recently, several optimal patterns for unbounded areas with special constraints, e.g., connectivity among nodes, are proposed in the area of wireless networking [1], [2], [3], [4], [19], [23], [24]. However, the deployment areas of wireless networks are always geographically bounded. In many cases, they cannot be approximated as an infinitely large plane, and it is a problem whether Kershner's pattern, as well as its following results, for infinitely large plane coverage can be directly applied to bounded areas. So careful studies on coverage of bounded areas is an imperative and of much significance for wireless networking.

Compared with the flourishing of research in unbounded area coverage, few results have been reported on how to optimally cover bounded areas. Many of these works are conjectural [15][16] or approximate [10]. To our knowledge, there have not been any theoretically guaranteed results on optimally covering bounded areas.

\footnotetext{
* The two authors are co-primary authors of this paper.
}

One reason for the slow progress in bounded area coverage research is that, for bounded areas, each single area and its boundary are unique. It is very hard to obtain any generic result or optimal pattern. It is not difficult to imagine that a little change in the boundary, e.g., pushing the boundary outward a little, may result in a total change of the optimal pattern. On the other hand, every point in the bounded area is unique. For infinitely large areas, every point can be considered equivalent, e.g., their Voronoi polygons are the same. Many results on optimal coverage of infinitely large areas depend on or require this feature. However, every point in the bounded area is unique. So we can no longer use the handy equivalence feature of points.

\section{B. Our Contributions}

In this paper, we have addressed the problem of bounded area coverage from both the theoretical and practical perspectives. We claim the following contributions:

1, Lower Bound Derivation: We have derived a tight lower bound on the number of nodes needed to cover a bounded area. We first develop a general lower bound for convex areas, and then we extend the study onto bounding concave and other irregular areas. These lower bounds provide a reference to judge how good a deployment pattern is.

2, Deployment Pattern Design: We have designed a set of general deployment patterns for bounded areas. We first investigate the development of deployment patterns for rectangular and rectangle-based areas, which are the most common deployment areas. Then we propose a general methodology for efficient coverage of other irregular areas. We show through analysis that the number of nodes needed for coverage by our patterns is very close to the lower bound. For example, to cover a $1 \mathrm{~km}$ by $1 \mathrm{~km}$ area with discs of radius 30 meters, our pattern needs only around $8 \%$ more nodes than the lower bound does.

3, Practical Considerations: We have also addressed other practical issues for deploying nodes to cover bounded areas. In the pattern design, we assume the coverage model is a disc, which is not always true in reality. We use the real-world trace to derive a more practical coverage model and study the performance of our proposed patterns. Besides coverage, we have shown that our designed patterns can achieve global connectivity among nodes when the communication range is properly large.

Paper Organization: The rest of the paper is organized as follows. Section II introduces related work and research on the coverage problem. Section III presents lower bound analysis 
of the needed nodes to cover bounded areas. In Section IV, we design deployment patterns for rectangular areas. In Section $\mathrm{V}$, we address node deployment in other areas than rectangles. Practical considerations on these patterns in realistic scenarios are discussed in Section VI. We conclude the paper with Section VII.

\section{RELATED WORK}

The optimal deployment pattern problem is fundamental. Related works are scattered throughout both the areas of geometry and wireless networks.

Directly relevant to the lower bound in our paper, there are several classical papers on the problem of how large an area $n$ congruent shapes can cover. Bamba et al. developed a bound on the largest area of a hexagon $H$ that can be covered (with simple intersection) by $n$ congruent convex domains $K$, i.e., $a(H) \leq n h(K)$, where $a(H)$ is the area of $H$ and $h(K)$ is the maximum inscribed hexagon area in $K$ [5]. G. F. Toth improved the result by adding a rectifying term on the right hand of the inequality [20]. Later Boroczky gave a (nearly) optimal bound of node density to cover hyperbolic planes [6]. As the bounds given by Boroczky is generic, it is not as tight as the bounds given by G. F. Toth in the research area of interest to us. However, none of them have used the results to study coverage of bounded areas. We have improved the results of Toth, and applied the the improved results to solving the bounded area coverage problem.

Some approximate patterns are proposed for covering certain bounded areas with specific shapes. Melissen et al. studied how to cover a bounded square with a small number of discs, i.e., 6-8 [15], and Nurmela et al. extended their work up to 30 discs in [16]. Their patterns are highly specific, i.e., a unit square can be optimally covered by $n$ discs with a specific radius. Hochbaum et al. provided an approximation scheme to cover orthogonal bounded rectangles in [10]. Though the scheme can provide a pattern infinitely close to optimality, the computation cost is prohibitively high, i.e., not polynomial regarding the approximation ratio $1 / \epsilon$.

There is also a large corpus of literature on covering infinitely large, i.e., unbounded, areas with discs, with or without other constraints. Kershner gave the most well-known result that the honeycomb structure, also known as the triangular lattice, is the optimal pattern to cover unbounded areas [12]. In the area of sensor networks, optimal deployment patterns to achieve coverage or connected coverage have been intensively studied. Bai et al. have reported several optimal regular patterns to achieve full coverage and different degrees of connectivity in two dimensional and three dimensional space [1], [2], [3], [4]. Yu et al. have designed optimal patterns for connected coverage in wireless networks with directional antennas [24]. Several works focus on how to select the minimum number of sensors to be activated from a set of randomly pre-deployed sensors such that all interested discrete locations (or targets) are $k$-covered. This problem is known to be NP-hard [23]. Centralized and distributed approximation algorithms were then proposed [9], [19], [23].
Another relevant category of research is that of optimally covering discrete points with sets [8]. It is a computation complexity and algorithm problem. In general, it is NP-hard, so only approximation algorithms are available [8]. In wireless networks, discrete points coverage in the unit disc graphs is much studied, as unit disc graphs comply with real wireless model and have many favorable features. An overview of this problem can be found in the literature review of [21], [22]. An important branch of the above problem which attracts much attention is to find a dominating point set in unit disc graphs. The latest results are reported by Zou et al. in [25]. As we are concerned in our problem about covering all the points of an area, these algorithms can only provide a very coarse estimate (often without theoretical guarantee) of the number of nodes needed for coverage of the entire area.

\section{LOWER BOUND ANALYSIS}

In this section, we present lower bound analysis of the number of nodes needed to cover a bounded area. We first present the main results along with the road map. Then we give the detailed proof of the results.

\section{A. Main Results and Road Map}

Instead of directly finding the minimum number of nodes to cover a bounded area, which is very difficult, we approach the problem from another angle. Suppose we want to cover a convex bounded area with discs. We ask the following question: What is the largest convex area that $n$ identical discs can cover? We will call this problem MaxCov hereafter. The answer to MaxCov gives an upper bound of how large an area $n$ discs can cover. In other words, given a convex bounded region of the same area, $n$ is the lower bound on the number of nodes needed to cover this area.

It is worth noting here that the convexity requirement in MaxCov targets more at confining the positions of the circles than at restricting the boundary shape. It is easy to see that the largest area $n$ discs can cover is $n$ times the area of one disc, when all the discs are placed well apart. But this kind of placement provides no coverage at all, as there are too many holes. So the convexity requirement is imposed here to rule out this possibility and force continuous coverage. Nonetheless, we will also study the cases of covering concave and other irregular areas later in this paper.

MaxCov is a classical problem, and has not been solved so far. The problem was studied in [5], [20]. The tightest bound given so far is the result in [20] dating back to 1987. In this section, we are going to improve this bound, and, hence, improve the lower bound of nodes needed to cover the area. In order to present our main results, we need to define some terminology first.

By a domain we mean a bounded closed set in the plane with interior points. We denote the disc of radius $r$ as $C$. We also denote the convex area to be covered as $K$, with boundary bd $K$. Their areas are represented by $a(C)$ and $a(K)$. Let $h(C)$ be the largest area of a hexagon contained in $C$, and $q(C)$ be the largest area of a quadrangle contained in $C$. Now 


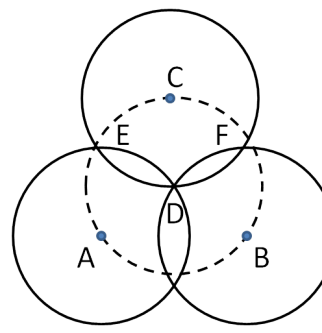

(a)

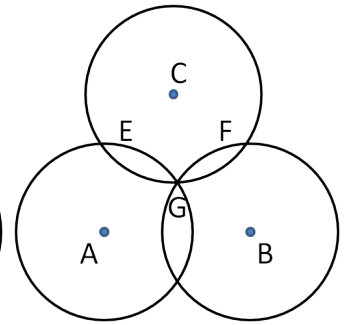

(b)

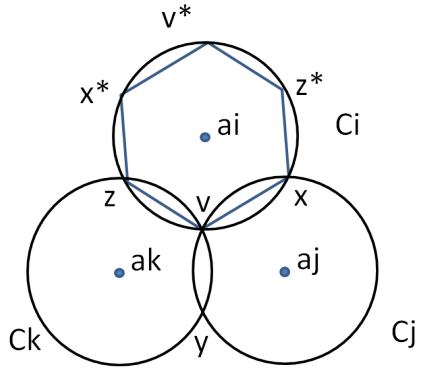

(c)

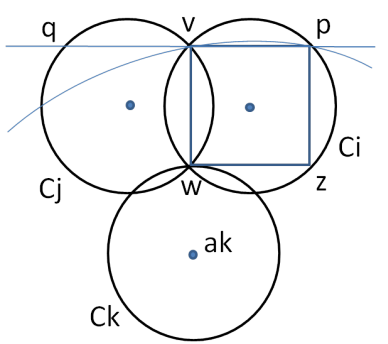

(d)

Fig. 1: Boundary areas where three circles intersect

we can present our main result Theorem 1, which gives a tight lower bound on the area $n$ identical convex domains $C$ can cover.

Theorem 1: If a convex domain $K$ is covered by $n \geq 2$ translates of a circle $C$, then $a(K) \leq(n-1) h(C)+a(C)-$ $\frac{\left(h^{*}+2 h(C)-2 a(C)\right)}{4(a(C)+q(C))}\left(\sqrt{d}-2 h^{*}+2 a(C)-4 h(C)-2 q(C)\right)$, where $d=\left(2 h^{*}-2 a(C)+4 h(C)+2 q(C)\right)^{2}-4(a(C)+$ $q(C))(-3 a(C)+4 h(C)-4 n h(C)+q(C))$ and $h^{*}=\left(24 \pi r^{2}-\right.$ $\left.\sqrt{35+\sqrt{73}}(2 \sqrt{2}+\sqrt{37-\sqrt{73}}) r^{2}\right) / 12$.

By conversely applying Theorem 1, we can get the lower bound. For example, if we decide that 968 discs with $r=1$ can at most cover an area of 2500.24 , then we know the minimum number to cover a $50 \times 50$ square is 968 . In fact, we will show that we can find a pattern with 1003 nodes to cover this square. 1003 is only $4 \%$ larger than the lower bound.

\section{B. Detailed Proof}

The derivation of this bound is based on a lemma which states that for maximum coverage, the coverage pattern should be trihedral, i.e., any vertices in the pattern are on the boundary of three discs. With this lemma, we can study the intersection areas of the three discs. Toth gave some criteria for maximum coverage in [20]. However, we have found out that some of the criteria cannot be satisfied at the same time. That is to say, the bound proposed in [20] is not reachable. Based on this observation, we derive the a tighter bound through careful calculations.

Let $D_{i}$ consists of those points $x$ of $K$ for which $f(x-$ $\left.a_{i}\right) \leq f\left(x-a_{j}\right)$ for all $j=1, \ldots, n$, where $f(\cdot)$ is the distance function. $D_{i}$ will be called a generalized Dirichlet cell associated with $a_{i}$. Let $D_{0}$ be the closure of the complement of $K$. The sets $D_{i}, i=0, \ldots, n$, constitute the faces of a cell complex $\varphi$. A point of the plane is said to be a vertex of $\varphi$ if it belongs to the boundary of at least three faces of $\varphi$. An edge of $\varphi$ is a connected component of the intersection of the boundaries of two faces in $\varphi$ containing more than one point.

Let $E$ and $V$ denote the set of edges and vertices of $\varphi$ respectively. With each edge $e \in E$ we associate a set $L(e)$ as follows: if $e$ is an edge common to the cells $D_{i}$ and $D_{j}$, and $i, j \neq 0$, then let $L(e)=C_{i} \cap C_{j}$. If on the other hand, $e$ is an edge common to $D_{i}$ and $D_{0}$, then let $L(e)$ be the connected component of $C_{i} \cap D_{0}$, the boundary of which contains $e$. For a vertex $v \in V$, in which the edges $e_{1}, e_{2}$ and $e_{3}$ meet, let $I(v)$ be the connected component of $L\left(e_{1}\right) \cap L\left(e_{2}\right) \cap L\left(e_{3}\right)$ which contains $v$. Let $t(v)=a\left(L\left(e_{1}\right)\right)+a\left(L\left(e_{2}\right)\right)+a\left(L\left(e_{3}\right)\right)-$ $2 a(I(v))$.

For a target area $K$ discussed in this section, we make the following five assumptions: (1) $C$ is a strictly convex domain with center of symmetry at the origin $O$; (2) Let $K$ be a convex domain, and $a_{1}, \ldots, a_{n}$ be distinct points such that $a_{i} \in K$ for $i=1, \ldots, n$; (3) For a point $x$ in the plane and any real number $\lambda, \operatorname{bd}(\lambda C+x)$ contains at most three of the points $a_{i}, i=1, \ldots, n$; and if $x \in \operatorname{bd} K$, then $\operatorname{bd}(\lambda C+x)$ contains at most two of the points; (4) $K \subset \bigcup_{i=1}^{n} C_{i}$, but no proper subset of $\left\{C_{i}\right\}_{i=1}^{n}$ covers $K$; (5) $K-C$ is connected.

It is proved in [20] that, if condition (3) is satisfied, then each vertex of the cell complex $\varphi$ is trihedral and $\varphi$ has exactly $2 n-2$ vertices. Now we present two lemmas regarding the coverage around these vertices. They are presented without proof due to space limitation.

Lemma 1: $a(K)=n a(C)-\frac{1}{2} \sum_{v \in V} t(v)$.

Lemma 2: Let $O_{A}, O_{B}, O_{C}$ and $O_{D}$ be four circles in Fig. 1(a) with $A, B$ and $D$ fixed. $O_{C}$ intersects with $O_{A}$ and $O_{B}$ at $E$ and $F$. Suppose the center of $O_{D}$ is $D$ and the center of $O_{C}$ lies on $O_{D}$. Then the $\left\|O_{A} \cap O_{C}\right\|+\left\|O_{B} \cap O_{C}\right\|$ is minimal when $C E \perp A B$.

Lemma 1 is about the overlapping areas around the vertices. Lemma 2 is on how to minimize the overlapping, so that the coverage area can be maximized. With these two lemmas, we are able to give the maximum area $n$ convex domains can cover by minimizing the overlapping around all the vertices. The result is presented in Theorem 2 .

Theorem 2: Let $b$ denote the number of vertices of $\varphi$ which are situated on bd $K$. Then we have

$$
\begin{aligned}
& a(K) \leq(n-1) h(C)+a(C)-\frac{1}{2} b\left(h^{*}+2 h(C)-2 a(C)\right), \\
& \text { where } h^{*}=\left(24 \pi r^{2}-\sqrt{35+\sqrt{73}}(2 \sqrt{2}+\right. \\
& \left.\sqrt{37-\sqrt{73}}) r^{2}\right) / 12 .
\end{aligned}
$$

Proof: Let $v$ be a vertex of $\varphi$ common to $D_{0}, D_{i}$ and $D_{j}$ (Fig 1d). We can see that $t(v)$ is the area of the part of the plane which is covered at least twice by $D_{0}, C_{i}$ and $C_{j}$. Looking for a lower power bounder for $t(v)$, we may suppose that the intersection $D_{0} \cap C_{i} \cap C_{j}$ consists of the single point $v$. Let $S$ be the closure of the complement of a supporting 
half-plane of $K$ such that $v \in$ bd $S$. Let $t^{*}(v)$ be the area of the part of the plane which is covered at least twice by $S$, $C_{i}$ and $C_{j}$. Then we have $t(v) \geq t^{*}(v)$. Let the second point of intersection of bd $C_{i}$ and $C_{j}$ be $w . w$ is an inner point of $K$ by assumption (v). It is not difficult to see that $t(w)$ is related to $t^{*}(v) . t(w)$ may be not minimal while $t^{*}(v)$ is. So we consider them as a whole next.

Let $O_{A}, O_{B}$ and $O_{C}$ be three circles in Fig. 1(b) with $A$ and $B$ fixed. $C G \perp A B$ and $O_{C}$ intersects with $O_{A}$ and $O_{B}$ at $E, F$ and $G$. Let $t^{*}(v)+t(w)=f(d)$, where $d=|A B|$.

Let $\angle A B G=\alpha$ and $\angle B C G=\beta$. Then

$f(d)=4\left(\frac{1}{2} \cdot 2 \alpha \cdot r^{2}-\frac{1}{2} \cdot \sin 2 \alpha \cdot r^{2}\right)+4\left(\frac{1}{2} \cdot 2 \beta \cdot r^{2}-\frac{1}{2}\right.$. $\left.\sin 2 \beta \cdot r^{2}\right)+2\left[\frac{1}{2}(\pi-2 \alpha) \cdot r^{2}-\frac{1}{2} \sin (\pi-2 \alpha) \cdot r^{2}\right]$

$=r^{2}\left[4 \alpha+2\left(\frac{\pi}{2}-\alpha\right)-2 \sin 2 \alpha-2 \sin \left(\frac{\pi}{2}-\alpha\right)+(\pi-2 \alpha)-\right.$ $\sin 2 \alpha]$

$$
\begin{aligned}
& =2 \pi r^{2}-\frac{3}{2} d \sqrt{4 r^{2}-d^{2}}-r \cdot d . \\
& f^{\prime}(d)=-r+\frac{3 d^{2}}{2 \sqrt{4 r^{2}-d^{2}}}-\frac{3 \sqrt{4 r^{2}-d^{2}}}{2}, \\
& f^{\prime \prime}(d)=\frac{3 d^{3}}{2\left(4 r^{2}-d^{2}\right)^{\frac{3}{2}}}+\frac{9 d}{2 \sqrt{4 r^{2}-d^{2}}} .
\end{aligned}
$$

$f^{\prime}(x)<0$ for each $x \in\left(0, \frac{\sqrt{35+\sqrt{73}}}{3 \sqrt{2}} r\right)$ and $f^{\prime}(x)>0$

for each $x \in\left(\frac{\sqrt{35+\sqrt{73}}}{3 \sqrt{2}} r, 2 r\right)$ since $f^{\prime}\left(\frac{\sqrt{35+\sqrt{73}}}{3 \sqrt{2}} r\right)=0$ and $f^{\prime \prime}(x)>0$ for each $d \in(0,2 r)$.

We have $t^{*}(v)+t(w) \geq\left(24 \pi r^{2}-\sqrt{35+\sqrt{73}}(2 \sqrt{2}+\right.$ $\left.\sqrt{37-\sqrt{73}}) r^{2}\right) / 12$ when $v \in$ bd $K$ by Lemma 2 . Let $h^{*}=$ $\left(24 \pi r^{2}-\sqrt{35+\sqrt{73}}(2 \sqrt{2}+\sqrt{37-\sqrt{73}}) r^{2}\right) / 12$.

When $v$ is a vertex of $\varphi$ common to $D_{i}, D_{j}$ and $D_{k}$ (Fig $1 \mathrm{c})$, where $i, j, k \neq 0 . t(v)$ is nothing else but the area of the part of the plane which is covered at least twice by $C_{i}, C_{j}$ and $C_{k}$. We deal it the same as in [20] for completeness. Similarly as above, we can assume that $v$ the only point common to $D_{i}$, $D_{j}$ and $D_{k}$ since we are looking for a lower bounder of $t(v)$. Then bd $C_{i}$ and bd $C_{j}$, bd $C_{j}$ and bd $C_{k}$, and bd $C_{i}$ and bd $C_{k}$ intersect at the points $x=a_{i}+a_{j}-v, y=a_{j}+a_{k}-v$ and $z=a_{k}+a_{i}-v$. Let $x^{*}, z^{*}$ and $v^{*}$ be the points obtained from $x, z$ and $v$. Then $x^{*}=a_{i}-a_{j}+v, z^{*}=a_{i}-a_{k}+v$, and $v^{*}=2 a_{i}-v$. Now, $x, z, v, x^{*},, z^{*}$ and $v^{*}$ are the vertices of a centrally symmetric convex hexagon $H$ inscribed in $C_{i}$. The sides $x z^{*}$ and $x^{*} z$ are parallel to the segment $v y$. This means that $t(v)=a(C)-a(H)$. Hence, $t(v) \geq a(C)-h(C)$ if $v \notin$ bd $K$. Similarly, when $v$ is a vertex of $\varphi$ common to $D_{0}, D_{i}$ and $D_{j}$, It follows $t(v) \geq a(C)-q(C)$, where $q(C)$ denotes the maximum area of a quadrangle contained in $C$.

For a vertex, $v$, of $\varphi$ common to $D_{0}, D_{i}$ and $D_{j}$. Let $w$ be the inner intersection point of $C_{i}$ and $C_{j}$. By the discussion in the first paragraph, we can see that, $t(v)+t(w) \geq\left(24 \pi r^{2}-\right.$ $\left.\sqrt{35+\sqrt{73}}(2 \sqrt{2}+\sqrt{37-\sqrt{73}}) r^{2}\right) / 12 \geq(a(C)-h(C))+$ $(a(C)-q(C))$.

Let $b$ denote the number of vertices of $\varphi$ which are situated on bd $K$. By Lemma 1 and the fact, $\varphi$ is trihedral and $\varphi$ has exactly 2 n-2 vertices, we have $a(K) \leq(n-1) h(C)+a(C)-$ $\frac{1}{2} b\left(h^{*}+2 h(C)-2 a(C)\right)$.

Theorem 2 is a very important intermediate result. It provides geometrical insight, as well as enabling comparison of our result against Toth's. We have two points to make regarding the comparison:

First, we notice that in [20], the main idea is to find the minimal area of the region covered by circles at least twice. All the vertices are divided into two cases: one kind of vertices are on the boundary of the convex area, and the other are inner points of the convex area. In [20], the minimal values of $t(v)$ are discussed independently. However, we can see that those $t(v)$ are related to each other. They can not achieve the minimum at the same time;

Second, the result in [20] is $a(K) \leq(n-1) h(C)+a(C)-$ $\frac{1}{2} b(h(C)-q(C))$. Since $h^{*} \geq(a(C)-h(C))+(a(C)-q(C))$, an easy calculation shows that $(n-1) h(C)+a(C)-\frac{1}{2} b\left(h^{*}+\right.$ $2 h(C)-2 a(C)) \leq(n-1) h(C)+a(C)-\frac{1}{2} b(h(C)-q(C))$. This means that the convex area which can be covered by given circles is strictly smaller than $(n-1) h(C)+a(C)-$ $\frac{1}{2} b(h(C)-q(C))$. Numerically, when $r=1$, Toth's result can be approximated by $3 \sqrt{3} n / 2-0.425 \sqrt{n}+0.878$, our result can be approximated by $3 \sqrt{3} n / 2-0.503 \sqrt{n}+0.946$. Ours is smaller than Toth's, when $n \geq 2$. Moreover, in the area close to the boundary, we have $\left[h^{*}+2 h(C)-2 a(C)\right] /[(h(C)-q(C)]>$ 1.18 when $r=1$. In other words, it needs at least $18 \%$ more nodes to cover the area close to the boundary a given convex area than [20].

We can further tighten the bounds in Theorem 2 with some algebraic treatment, which leads to our main result Theorem 1. Here comes the proof of Theorem 1

Proof: It is obtained in [20] that $a(K) \leq\left(\frac{b+1}{2}\right)^{2}(a(C)+$ $q(C))$. By Theorem 2, it is easy to check that $\left(\frac{b+1}{2}\right)^{2}(a(C)+$ $q(C)=(n-1) h(C)+a(C)-\frac{1}{2} b\left(h^{*}+2 h(C)-2 a(C)\right)$ when $b=b_{0}=\frac{1}{2(a(C)+q(C))}\left(\sqrt{d}-2 h^{*}+2 a(C)-4 h(C)-2 q(C)\right)$, where $d=\left(2 h^{*}-2 a(C)+4 h(C)+2 q(C)\right)^{2}-4(a(C)+$ $q(C))\left(-3 a(C)+4 h(C)-4 n h(C)+q(C)\right.$ and $h^{*}=\left(24 \pi r^{2}-\right.$ $\left.\sqrt{35+\sqrt{73}}(2 \sqrt{2}+\sqrt{37-\sqrt{73}}) r^{2}\right) / 12$.

For $b<b_{0}, a(K) \leq\left(\frac{b+1}{2}\right)^{2}(a(C)+q(C))$, for $b>$ $b_{0}, a(K) \leq(n-1) h(C)+a(C)-\frac{1}{2} b\left(h^{*}+2 h(C)-\right.$ $2 a(C))$ is stronger than $a(K) \leq(n-1) h(C)+a(C)-$ $\frac{\left(h^{*}+2 h(C)-2 a(C)\right)}{4(a(C)+q(C))}\left(\sqrt{d}-2 h^{*}+2 a(C)-4 h(C)-2 q(C)\right)$.

\section{Deployment Pattern Design for RECTANGULAR AREAS}

In this section, we design deployment patterns to cover a large category of common bounded areas, i.e., the target area $K$ is a rectangle. Rectangular areas are very common in practice, and most realistic deployment areas can be decomposed into or approximated by rectangular areas. We then compare the performance of the pattern with the lower bound, and show that the patterns can cover rectangular areas very efficiently.

When designing deployment patterns for bounded rectangular areas, we borrow the insight from the optimal pattern to cover infinitely large space, i.e., using the honeycomb structure. The idea here is that to cover the points not close to the boundary, the optimal deployment pattern is likely to resemble the infinitely large area case to a large extent. However, things are different near the boundary. We need to carefully adapt the honeycomb pattern to handle the boundary. 
One important consideration here is to precisely position the honeycomb structure so that there are as few 'wasted' or unnecessary cells on the boundary as possible.

As stated above, we use the honeycomb deployment pattern (the background structure in Fig 2). The covering discs are the circumscribing circle of those hexagonal cells. The discs have a radius of 1 unit length. In other words, the honeycomb structure is the Voronoi diagram of the deployment pattern. We can think of the honeycomb structure as superposed on the rectangular area. It is not necessarily aligned so well along the borders of the rectangle as is shown in Fig. 2. In fact, it can shift randomly. We pick all the cells that have intersection with the area to be covered. We call the collection of these cells the Random Honeycomb Pattern (RHP) over the area to be covered. RHP is a naive deployment directly adapted from the infinitely large case. Apparently, it can perform poorly due to lack of careful positioning. Take the case in Fig. 2 for example, if the honeycomb structure is moved rightward a little, we will need an extra column on the left border to cover the area. So careful calibration of the honeycomb structure can lead to much better performance.

In order to position the structure properly, we first need to separate the honeycomb structure into repeatable and disjoint 'tile'. An example of the tile is the small rectangle at the lower left corner in Fig. 2. Shifting the RHP one whole tile in any direction results in the same pattern. So it makes little sense to move over one tile in length or in height. In fact, the small rectangle in Fig. 2 is the smallest tile that contains one entire cell. So it provides us the longest distance to meaningfully shift the honeycomb, i.e., no more than $\sqrt{3}$ unit lengths vertically and no more than 3 horizontally.

The tile positioning as is illustrated in Fig. 2 ensures fewest unnecessary cells on the boundary. Just look at the left and bottom border. Intuitively if we move the whole honeycomb structure rightward or upward a little bit, we will need extra cells to cover the left and the bottom border of the rectangle. If we move it leftward or downward a little bit, we waste the covering capacity of the cells on the left and bottom border. So this positioning achieves minimum waste on the left and bottom border. Of course, we want this type of perfect positioning and minimum waste to happen on all four borders of the whole area. It is not always possible, because the lengths of $|A D|$ and $|A B|$ are already specified. So at most we can ensure there are no 'wasted' cells on two borders out of four. In this light, the pattern shown in Fig. 2 is the best tile positioning we can hope to achieve. And the aligned version of the RHP is the deployment pattern we propose.

To count the number of nodes needed for the proposed pattern in Fig. 2, there are several different cases to consider. Suppose $|A B|=a$ and $|A D|=b, \mid a / \sqrt{3}\rfloor=m$ and $\lfloor b / 3\rfloor=n$. For width $|A B|$, we consider two cases (Fig 2). Case 1: $B$ is between $v_{0}$ and $v_{1}$; Case 2: $B$ is between $v_{1}$ and $v_{2}$. For length $|A D|$, we have three cases to consider. Case 1: $D$ is between $h_{0}$ and $h_{1}$; Case 2: $D$ is between $h_{1}$ and $h_{2}$; Case 3: $D$ is between $h_{2}$ and $h_{3}$. Now we will calculate the number of nodes needed to cover $K$.

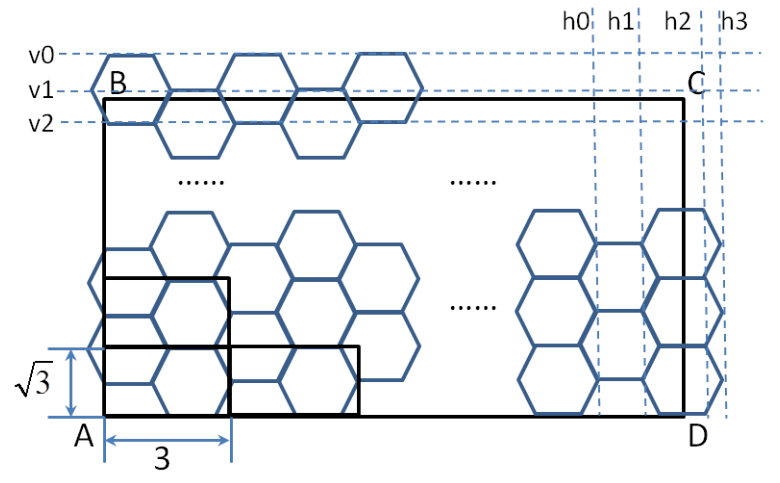

Fig. 2: The honeycomb structure

Case 1: $B$ is between $V_{0}$ and $V_{1}$ and $D$ is between $h_{0}$ and $h_{1}$, i.e., $\sqrt{3} m<a \leq \sqrt{3}(m+0.5)$ and $3 n<b \leq 3 n+1$. The number of nodes we need is $(m+1)(2 n+1)$.

Case 2: $B$ is between $V_{0}$ and $V_{1}$ and $D$ is between $h_{1}$ and $h_{2}$, i.e., $\sqrt{3} m<a \leq \sqrt{3}(m+0.5)$ and $3 n+1<b \leq 3 n+5 / 2$. The number of nodes we need is $2(m+1)(n+1)$.

Case 3: $B$ is between $V_{0}$ and $V_{1}$ and $D$ is between $h_{2}$ and $h_{3}$, i.e., $\sqrt{3} m<a \leq \sqrt{3}(m+0.5)$ and $3 n+5 / 2<b \leq 3 n+3$. The number of nodes we need is $(m+1)(2 n+3)$.

Case 4: $B$ is between $V_{1}$ and $V_{2}$ and $D$ is between $h_{0}$ and $h_{1}$, i.e., $\sqrt{3}(m+0.5)<a \leq \sqrt{3}(m+1)$ and $3 n<b \leq 3 n+1$. The number of nodes we need is $(2 m+3) n+m+1$.

Case 5: $B$ is between $V_{1}$ and $V_{2}$ and $D$ is between $h_{1}$ and $h_{2}$, i.e., $\sqrt{3}(m+0.5)<a \leq \sqrt{3}(m+1)$ and $3 n+1<b \leq$ $3 n+5 / 2$. The number of nodes we need is $(2 m+3)(n+1)$.

Case 6: $B$ is between $V_{1}$ and $V_{2}$ and $D$ is between $h_{2}$ and $h_{3}$, i.e., $\sqrt{3}(m+0.5)<a \leq \sqrt{3}(m+1)$ and $3 n+5 / 2<b \leq$ $3 n+3$. The number of nodes we need is $(2 m+3)(n+1)+$ $m+1$.

We have conducted numerical evaluations on the coverage performance of our patterns against the lower bounds on several typical deployment areas. The results are shown in Fig. 3 and Fig. 4. We consider two typical types of rectangles for illustration. In Fig. 3, we consider squares. In Fig. 4, we consider rectangles with length equal to two times the width. Subfigure (a) of both plots show the number of discs needed to cover the area. The red solid line indicates the lower bound, the blue dashed line indicates our carefully positioned pattern, and the green dot-dashed line indicates the average performance of RHP. From subfigure (a), we can see that the three lines almost overlap. It means that these patterns are quite close in performance. In order to take a close look at the percentage of more nodes needed than the lower bound, we draw subfigure (b). The extra solid black line gives the worst case performance of RHP as a reference. First we observe that the lines are not smooth. It is because our designed patterns and RHPs have mutations across the cases we just discussed above. We can see that when the radius is smaller than 50 meters, our patterns need at most $12 \%$ more nodes than the lower bound. In comparison, the worst case almost doubles this percentage, which indicates its inefficiency. In average RHP needs around $18 \%$ more nodes than the lower bound when the disc radius is 
50 meters, $50 \%$ more extra nodes than our proposed pattern.

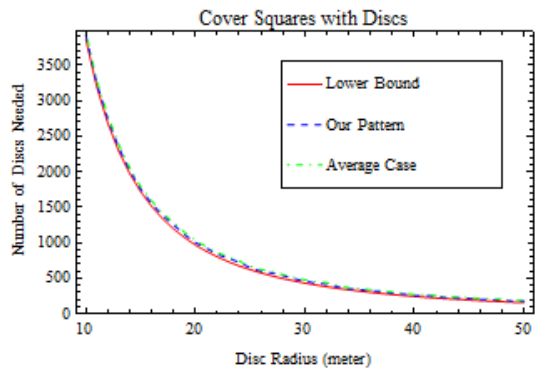

(a)

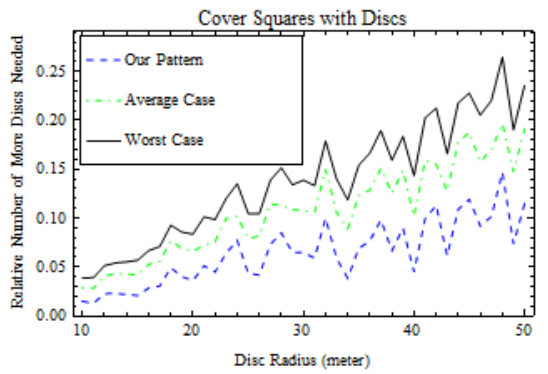

(b)

Fig. 3: (a) and (b): covering an $1 \mathrm{~km} \times 1 \mathrm{~km}$ area with discs of varying radii.

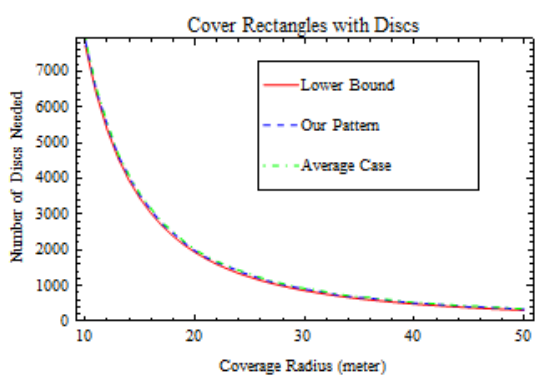

(a)

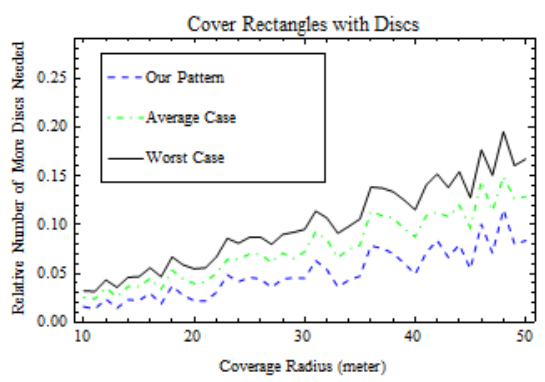

(b)

Fig. 4: (a) and (b): covering a $2 \mathrm{~km} \times 1 \mathrm{~km}$ area with discs of varying radii.

Before ending this section, we want to clarify one point. We only consider moderately large areas in Fig. 3 and Fig. 4 , and do not consider extremely small areas, e.g., a square deployment area with side length equal to 2 or 3 times the disc diameter. The reason is as follows. As one of the first

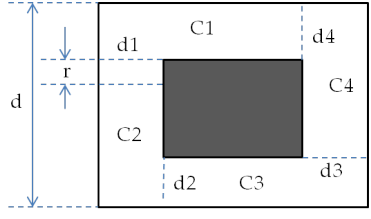

(a)

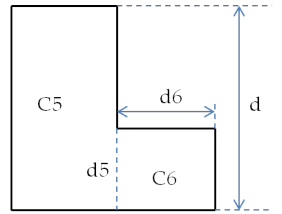

(b)
Fig. 5: Two types of rectangle-based areas we are going to study, (a) rectangular band and (b) L-shape or rectangle-based art gallery shape

attempts to address the problem of covering bounded areas, we want to give a big picture and provide as generic results as possible. This mission is possible for moderately large areas, and is both unnecessary and extremely difficult for extremely small areas. For extremely small areas, it is indeed a much better idea to study each specific area shape independently. The extremely small area makes each covering disc so unique that almost any generalization attempt will be defeated. In fact, the lower bound result proposed in the last section does not work well with this type of areas. However, the small area makes possible certain types of numerical analysis. The quasi-Newton method [16] and the approximation deployment method [10] are two feasible ways to find a good approximation pattern. Or if discretization of space is allowed, the minimum set cover method can be used to find the least number of nodes needed, as was done in [22]. These methods work for small areas, but as the number of discs needed grows, e.g., $>100$, the computation complexity will soon become prohibitively high. So analytical results like ours are needed to guide deployment.

\section{Deployment Pattern Design for GENERAL BOUNDED AREAS}

In this section, we are going to study the heuristic deployment pattern for more general bounded areas. We start from two relatively easy cases, i.e., the shapes illustrated in Fig. 5. The two shapes in the figure are square rings and L-shapes. They are extensions to the rectangular areas, and they can be often seen in everyday monitoring scenarios, e.g., perimeter security and art gallery surveillance. With the insight derived from the pattern design for these two rectangle-based areas, we will provide a general methodology and pattern for other more irregular deployment areas.

In Fig. 5, as the figures are not convex any more, the lower bound derviced in Section III is no longer directly applicable. On the other hand, we cannot directly apply the pattern designed in the last section for rectangles, because the shape of boundary changes. So we need derive a new lower bound, as well as a new set of deployment patterns.

We observe that though the areas in Fig. 5 is not rectangular, we can still decompose them into rectangular components. We can calculate the lower bound of the needed disc number to cover each of the components. However, some discs covering one component may 'stretch' into the neighboring component. So for a rigorous lower bound, we need to deduct that area from the neighboring component. Take an example, in Fig. 
5(a), discs covering $C_{1}$ may 'extend' into $C_{2}$, and cover a part of $C_{2}$. So we deduct a band of width $r$, the disc radius, in $C_{2}$ close to the cutting boundary to exclude the influence of those discs. Suppose $n($ Area $)$ is a lower bound of needed discs to cover Area. Then $n\left(C_{1}\right)+n\left(C_{2}-S\right)$ is a lower bound of needed disc number to cover the combined area of $C_{1}$ and $C_{2}$. Here $S=d_{1} \cdot r$. After cutting the rectangle-based shapes into rectangles, we can apply the deployment patterns proposed in the last section to each rectangle. We show the coverage performance results in Fig. 6 and Fig. 7. In Fig. 6, we consider a square with a hole which is a square of width equal to one half the outer square width, and placed in the center, i.e., $d_{1}=d_{2}=d_{4}=d_{4}=\frac{1}{4} d$ in Fig .5. In Fig. 7, we consider a specific L-shaped area in Fig. $5 . C_{5}$ is a rectangle with length equal to two times the width, and $C_{6}$ is a square with the same width, i.e., $d_{5}=d_{6}=\frac{1}{2} d$. This shape can be imagined as a larger square with its upper right $1 / 4$ part removed. The coverage performance after applying our deployment patterns is given in Fig. 6 and Fig. 7, which shows similar features to Fig. 3 and Fig. 4. It is worth noting that the performance is a little worse than in pure rectangle coverage cases, as the deployment areas are more irregular.

In real world deployment, we may encounter many areas far more irregular. It is hard to design optimal patterns for each of them. However, we can borrow the ideas from covering rectangular and rectangle-based areas. Basically, we want to reduce the area to 'manageable' shapes, such as regular shapes. Then we can carefully position the honeycomb structure to cover the manageable shapes. Here we give some discussions on how to achieve this. This is, in fact, a part of the future work of this paper.

First, we discuss how to construct the building block, i.e., covering 'manageable' areas, mainly convex polygonal areas similar to regular shapes. The basic idea of the deployment pattern is again to use the honeycomb structure. We can carefully position the honeycomb structure and minimize the waste on boundaries. For regular areas, like regular triangles or hexagons, we can indeed decide the optimal positioning by analyzing the boundary cells. For other less regular areas, we can at least try to achieve optimal covering on as many sides as possible.

For non-convex and irregular areas, we approximate them and decompose them into the manageable areas described above. One example of the irregular area is shown in Fig. $8(\mathrm{a})$, we need to find a tightly circumscribing polygonal area like Fig. 8(b) for pattern design and a tightly inscribed polygonal area like Fig. 8(c) for lower bounding. The approximate decomposition can be efficiently performed using the algorithms proposed in [14]. Then we design patterns, either RHP or its variant, to cover all the subareas in the circumscribing polygonal area. The needed node number can serve as an upper bound. If this upper bound is close to the lower bound, then this pattern achieves efficient coverage. On the other hand, we use the inscribed polygonal area to calculate the lower bound. As noted earlier in this section, in order to satisfy the definition of lower bound, we need

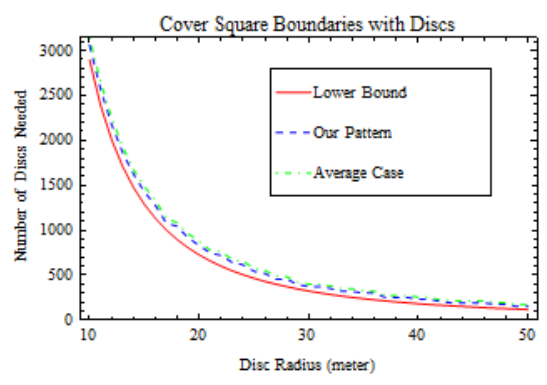

(a)

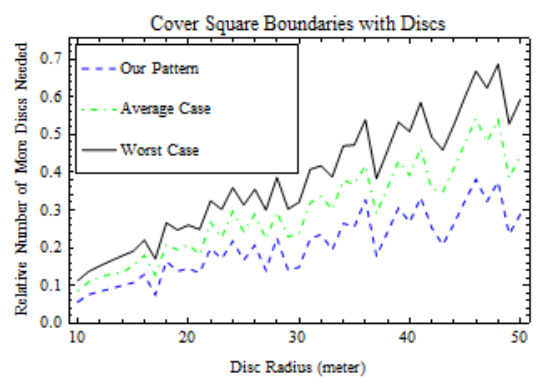

(b)

Fig. 6: (a) and (b): covering a square boundary (outer square width is $1 \mathrm{~km}$ ) with discs of varying radii.

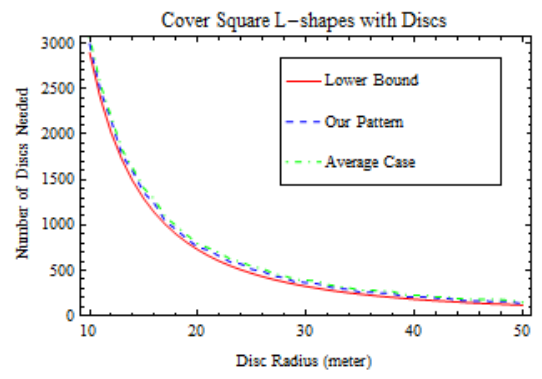

(a)

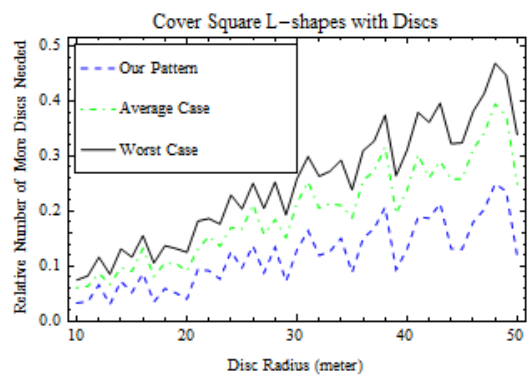

(b)

Fig. 7: (a) and (b): covering an L-shaped area (one side is $1 \mathrm{~km}$ ) with discs of varying radii.

to deduct a band of width $r$, the radius of disc, along the cutting boundary in at least one convex sub-polygon near the decomposition boundary. This band may be covered by discs on the other side of the boundary. In most cases, the deducted part can be approximated by $r \times d$, where $d$ is the total length of the cutting boundary. In this sense, we need to find the optimal polygon convex decomposition in terms 


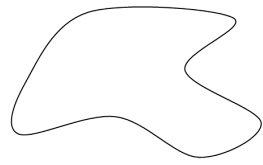

(a)

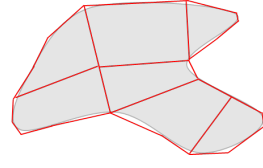

(b)

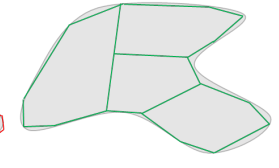

(c)
Fig. 8: Pattern design for complicated polygonal areas

of the boundary number, because the fewer boundaries, the more likely that our lower bound is tight. We can refer to [7] on optimal convex decomposition. It is even better if we can improve this method by requiring that the total length of the added boundary lines is minimum, because the number of discs to cover the boundary which are subtracted for lower bounding can be minimized in proportion to the boundary length. This kind of decomposition is doable if we do not use Steiner points [11], but only approximation algorithms are available for decomposition with Steiner points [13].

\section{Practical Considerations}

So far we have studied the problem of using discs to cover bounded areas. However, in real world deployment, we may encounter non-disc model. Also sometimes coverage is not the sole requirement. We may need connectivity between nodes. In this section, we address these practical issues.

\section{A. Non-disc Coverage Model}

In this subsection, we evaluate our patterns in a real world wireless environment. That is to say, we use our proposed patterns to cover certain bounded areas, but employ realistic coverage range model to see how well the patterns perform.

In a real world environment, the coverage range is normally not a disc and is changing over time due to environmental noises. We model the coverage range based on an urban area real trace. The trace we use is MetroFi [17]. MetroFi is a dataset of location, signal strength (RSSI or Received Signal Strength Indicator), and performance data of the MetroFi network. This network is an $802.11 \mathrm{x}$ municipal wireless mesh network deployed in Portland, Oregon. The dataset was collected in 2007. It involves 72 Access Points (APs) that are deployed across the city, and the RSSI samples are taken at over 200,000 locations. We consider such a dataset representative for the wireless environment in a city-wide area.

We mine the trace and extract the coverage model in two ways. In a simple way, we consider a uniform AP model (virtualAP). The coverage radius of each AP is the same. Their RSSI distributions over distance follow the general lognormal path attenuation model with noise [26]. We derive the parameters of the path attenuation and noise based on curve fitting of the MetroFi dataset. The radius of our APs, $r$, is designed to guarantee a packet reception rate (PRR) of $90 \%$ on the circle boundary. In the second model, we consider a more practical model: every AP has a different RSSI distribution in its domain (realAP). Each specific AP model is randomly selected from the 72 APs in the MetroFi dataset. The whole coverage area is a rectangle with width and length equal to 20r. According to our pattern, we need 175 APs to cover this area. It is worth noting that in real deployment, there are probably more wireless nodes to cover an area than necessary for robustness. There will be some scheduling mechanism such as [18] to keep a part of them awake and another part dormant. Our designed pattern and the following evaluations can help determine the number of active nodes.

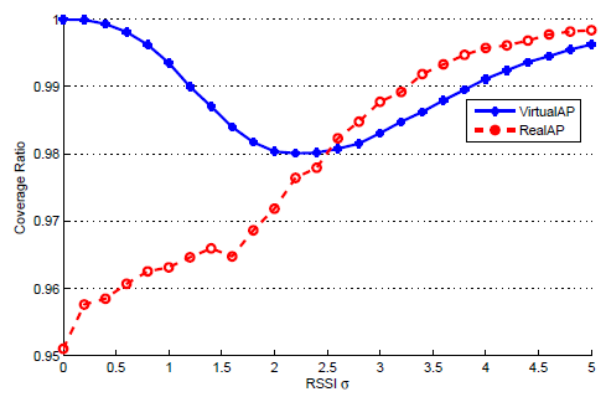

(a)

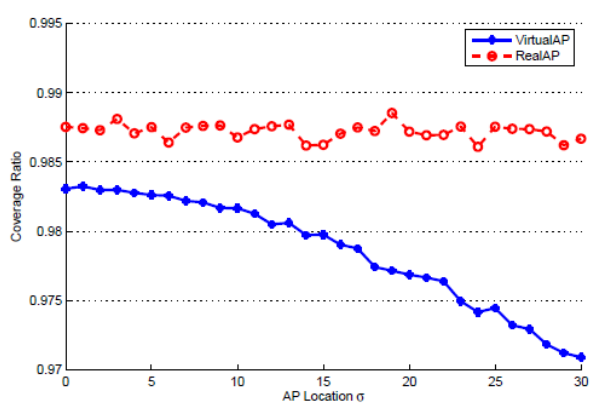

(b)

Fig. 9: Coverage Performance in Real Networking Scenarios

Fig. 9a evaluates the coverage when there are different levels of noise in the environment, when the APs are deployed according to our designed patterns. The intensity of noise is measured by the RSSI standard deviations. Fig. $9 \mathrm{~b}$ evaluates the coverage performance when the node deployment locations are not as accurate as in our pattern, i.e,, they have a random shift with standard deviation $\sigma$. It may be caused by random deployment errors or existence of obstacles. We make the following observations. First, the coverage ratio of virtualAP decreases as the location deviation increases, because the real deployment is farther and farther away from our designed patterns. Second, the coverage ratio of virtualAP decreases first and then increases as the RSSI deviation increases. The increase in the second part can be explained by the enhanced influence of neighboring APs, which helps to increase the coverage. Third, the coverage ratio of realAP is more robust against both deviations. It keeps stable or even increases with larger deviations. This is because the RSSI distributions of each AP are different and the coverage shapes are diverse, which fill the coverage gaps brought by the deviations.

\section{B. Connectivity}

In many types of wireless networks, coverage is not the only requirement. Connectivity among nodes is also vital. In 
WSNs, sensor nodes need to be connected to forward messages to the sink node. In wireless mesh networks (WMNs), APs need to connect to each other to form the wireless backbone of the whole network. Sometimes, these WMN APs will use a different radio to form the backbone. So the APs may have two wireless transmission ranges, one for covering local clients and the other for communicating with the backbone networks. In this subsection, we discuss the connectivity issue related to our patterns, when wireless connected coverage is required.

As was said in Section I, bounded areas may see very different boundaries. So it is not surprising that generic results about connectivity are very hard to derive. However, we may still reach some conclusions, when the communication range, $r_{c}$, is over $\sqrt{3}$ times the coverage range, $r$, i.e., $r_{c} \geq \sqrt{3} r$. It is normally a reasonable requirement to ensure that the communication range is appropriately large. Because, for connected coverage of an area, inappropriately small communication range will result in a large waste of the node coverage capability. We consider the pattern of randomly covering a convex area with the honeycomb structure, i.e., the Random Honeycomb Pattern (RHP). All the deployment patterns we have proposed above can be derived from this pattern. Regarding RHP, we present Theorem 3 without proof due to space limitation.

Theorem 3: When $r_{c} \geq \sqrt{3} r$ and the covered area is convex, the Random Honeycomb Pattern is connected. Under the same condition, the Random Honeycomb Pattern is 2connected, if it is not a single line deployment pattern.

It is not difficult to see that all the deployment patterns we have proposed above is a variation or development of the RHP pattern. The deployment patterns for the convex shapes are a direct application and optimization of this pattern. The deployment patterns of those concave shapes can be separated into several such patterns covering (nearly) convex areas. On the cutting boundaries of the concave shapes, there are at least as many covering cells as required. So the connectivity performance there is better than the simple honeycomb structure. So Theorem 3 holds for almost all the patterns we have proposed.

\section{CONCLUSION}

In this paper, we investigated the problem of how to optimally cover a bounded area with discs. We have derived a theoretical lower bound of the node number to cover bounded areas. Based on this result, we have proposed several deployment patterns for different kinds of deployment area shapes. Numerical analysis and trace-based evaluations have proved that our deployment patterns are close to optimal and robust in realistic scenarios.

\section{ACKNOWLEDGMENT}

The work was supported in part by China 973 Project 2011CB302800, the National Science Foundation of China (NSFC) under grant No. 61070221, 61070245, and the US National Science Foundation (NSF) under Grant No. CNS0916584, CNS1065136 and CNS1218876. Any opinions, findings, conclusions, and recommendations in this paper are those of the authors and do not necessarily reflect the views of the funding agencies.

\section{REFERENCES}

[1] X. Bai, S. Kumar, D. Xuan, Z. Yun, and T. Lai. Deploying wireless sensors to achieve both coverage and connectivity. In Proc. of ACM MobiHoc, 2006

[2] X. Bai, D. Xuan, Z. Yun, T. Lai, and W. Jia. Complete optimal deployment patterns for full-coverage and k-connectivity $(\mathrm{k} \leq 6)$ wireless sensor networks. In Proc. of ACM MobiHoc, 2008.

[3] X. Bai, Z. Yun, D. Xuan, B. Chen, and W. Zhao. Optimal multiplecoverage of sensor networks. In Proc. of IEEE INFOCOM, 2011.

[4] X. Bai, C. Zhang, D. Xuan, J. Teng, and W. Jia. Low-connectivity and full-coverage three dimensional wireless sensor networks. In Proc. of ACM MobiHoc, 2009.

[5] R. P. Bambah and C. A. Rogers. Covering the plane with convex sets. J. London Math. Soc, 27:304-314, 1952.

[6] K. Boroczky. Finite coverings in the hyperbolic plane. Discrete \& Computational Geometry, 33:165-180, 2005.

[7] B. Chazelle and D. P. Dobkin. Optimal convex decompositions. Computational Geometry, pages 63-133, 1985.

[8] T. Gonzalez. Covering a set of points in multidimensional space. Information Processing Letters, 40(4):181-188, 1991.

[9] M. Hefeeda and M. Bagheri. Randomized k-coverage algorithms for dense sensor networks. In Proc. of IEEE INFOCOM, 2007.

[10] D. Hochbaum and W. Maass. Approximation schemes for covering and packing problems in image processing and VLSI. Journal of the ACM, 32(1):130-136, 1985.

[11] J. M. Keil. Decomposing a polygon into simpler components. SIAM J. Comput., 14(4):799-817, 1985.

[12] R. Kershner. The number of circles covering a set. American Journal of Mathematics, 61:665-671, 1939.

[13] C. Levcopoulos and A. Lingas. Bounds on the length of convex partitions of polygons. In Proc. 4th Conf. Found. Softw. Tech. Theoret. Comput. Sci., volume 181, pages 279-295. Lecture Notes Comput, Springer-Verlag, 1984.

[14] J.-M. Lien and N. M. Amato. Approximate convex decomposition of polygons. In Proc. of ACM SCG, pages 17-26, 2004.

[15] J. Melissen and P. Schuur. Improved coverings of a square with six and eight equal circles. Electronic Journal of Combinatorics, 3(1), 1996.

[16] K. Nurmela and P. Ostergard. Covering a square with up to 30 equal circles. Research Report A62, Laboratory for Theoretical Computer Science, Helsinki University of Technology, 2000.

[17] C. Phillips and R. Senior. CRAWDAD data set pdx/metrofi (v. 201110-24). Downloaded from http://crawdad.cs.dartmouth.edu/pdx/metrofi, Oct. 2011.

[18] S. Ren, Q. Li, H. Wang, X. Chen, and X. Zhang. Analyzing object detection quality under probabilistic coverage in sensor networks. In IWQoS, pages 107-122, Passau, Germany, June 2005.

[19] G. Simon, M. Molnar, L. Gonczy, and B. Cousin. Dependable kcoverage algorithms for sensor networks. In Proc. of IMTC, 2007.

[20] G. Toth. Finite coverings by translates of centrally symmetric convex domains. Discrete Comput Geom, 2:353-363, 1987.

[21] P. Wan, X. Xu, and Z. Wang. Wireless coverage with disparate ranges. In Proc. of ACM MobiHoc, 2011.

[22] X. Xu and Z. Wang. Wireless coverage via dynamic programming. In Wireless Algorithms, Systems, and Applications, volume 6843 of Lecture Notes in Computer Science, pages 108-118. Springer Berlin / Heidelberg, 2011.

[23] S. Yang, F. Dai, M. Cardei, and J. Wu. On connected multiple point coverage in wireless sensor networks. Journal of Wireless Information Networks, May 2006.

[24] Z. Yu, J. Teng, X. Bai, D. Xuan, and W. Jia. Connected coverage in wireless networks with directional antennas. In Proc. of IEEE INFOCOM, 2011.

[25] F. Zou, Y. Wang, X.-H. Xu, X. Li, H. Du, P. Wan, and W. Wu. New approximations for minimum-weighted dominating sets and minimumweighted connected dominating sets on unit disk graphs. Theoretical Computer Science, 412(3):198-208, 2011.

[26] M. Zuniga and B. Krishnamachari. Analyzing the transitional region in low power wireless links. In Proc. of SECON, pages 517-526, 2004. 